\title{
Ethanol Metabolism
}

National Cancer Institute

\section{Source}

National Cancer Institute. Ethanol Metabolism. NCI Thesaurus. Code C18221.

Ethanol Metabolism consists of various biochemical detoxification reactions that convert ethanol to metabolic derivatives for elimination from the body. 